\title{
Using perioperative multimodal analgesia results in decrease acute but not chronic postoperative pain in patients with lumbar herniated disc
}

Genov P.: ${ }^{1}$, Timerbaev V. ${ }^{1}$, Grin A. ${ }^{2}$, Rebrova O. ${ }^{3}$, Vyatkin A. ${ }^{1}$

${ }^{1}$ N.V. Sklifosovsky Scientific Research Institute for Emergency Medicine of Health Department of Moscow, Dept of Anaesthesiology, Moscow, Russian Federation; ${ }^{2}$ N.V. Sklifosovsky Scientific Research Institute for Emergency Medicine of Health Department of Moscow, Neurosurgery, Moscow, Russian Federation; ${ }^{3}$ Pirogov Russian National Research Medical University, Medical Cybernetics and Informatics, Moscow, Russian Federation.

\begin{abstract}
Background and Goal of Study: Using perioperative multimodal analgesia has a potency for prevention chronic postoperative (PO) pain. Failed back surgery syndrome (FBSS) is the debilitating chronic pain condition. Is it possible to decrease the rate of FBSS by improving the perioperative analgesia in patients with lumbar herniated disc?
\end{abstract}

Materials and methods: 129 patients (18-70 years, 2-3 ASA) scheduled for elective lumbar discectomy in 2010-2013 were enrolled in prospective observational study. GA+OD $(n=20)$ underwent general anaesthesia $(G A)$ and $P O$ analgesia on-demand. SB+PMA $(n=23)$ got subarachnoid block (SB) and preventive multimodal analgesia (PMA) including ketoprofen, paracetamol and nalbuphine on-demand. At GA+PMA ( $n=21) \mathrm{GA}$ and PMA were used; $G A+P M A+I(n=21)$ also had bupivacaine wound infiltration; at $G A+P M A+S$ $(n=20)$ - depo-corticosteroid was applied on affected spinal nerve root; at $\mathrm{GA}+\mathrm{PMA}+\mathrm{IS}(\mathrm{n}=24)$ wound infiltration and local corticosteroids were combined. 7 days PO the dinamic VAS pain scores were recorded. In 6 months the phone survey was performed and follow-up (NRS pain scores, quality of life (QOL), disability, sleep disorder) was assessed.
Results: As shown in Table 1, GA+OD had not adequate pain relief during $4 \mathrm{PO}$ days but $\mathrm{GA}+\mathrm{PMA}$ had. The VAS scores in SB+PMA were less than in GA+PMA only in 2 hours PO $(1(0-3,5)$ and $4(2,5-6)$, $p=0,002$, Mann-Whitney test). The VAS scores in $G A+P M A+I$ and $G A+P M A+I S$ were significant less, than in $G A+P M A$ throughout 2 PO days.

In 6 months $60 \%$ patients experienced back or leg pain and $30 \%$ - leg pain. Some patients suffered from FBSS with sleep disorder (24\%), disability (25\%), decrease QOL (23\%). There was not significant difference between groups in term of neither rate of chronic pain in common nor leg pain specifically $\left(p=0,459\right.$ and $p=0,903$ respectively, $x^{2}$ test) nor NRS pain scores ( $p=0,112$, Kruskal-Wallis ANOVA).

Conclusions: PMA is the better choice than analgesia on-demand. Additional bupivacaine wound infiltration follows significant pain relief during $2 \mathrm{PO}$ days. The rate of FBSS doesn't depend on the mode of perioperative analgesia.

Table 1. Postoperative pain scores, Me (LQ-UQ)

\begin{tabular}{|l|l|l|}
\hline 2nd POD, VAS & 6 months, \\
\hline $5(4-7)$ & $5(2,5-6)$ & $3(2-5)$ \\
\hline $3(1,25-4,5)^{\star}$ & $2(0,5-4)^{\star}$ & $2(1-3)$ \\
\hline $3(2-4,5)$ & $2,25(1-5)$ & $3(2-5)$ \\
\hline $1,5(1-3) \dagger$ & $1(0,5-2)$ & $3(3-3)$ \\
\hline $2,5(1-4)$ & $2(1-2,75)$ & $3(2-3)$ \\
\hline $1,75(0,75-3,25) \dagger$ & $1(0,25-3,5)$ & $3(1,5-3,75)$ \\
\hline
\end{tabular}

